\title{
Electronic Structure and Magnetic Moments of Copper-atom in/on GaN Semiconductor
}

\author{
Byung-Sub Kang ${ }^{1 *}$ and Haeng-Ki Lee ${ }^{2}$ \\ ${ }^{1}$ BK 21 Physics program and Department of Physics, Chungbuk National University, Cheongju 361-763, Korea \\ ${ }^{2}$ Department of Radiotechnology, Daegu Polytechnic College, Daegu 706-711, Korea
}

(Received 8 March 2010, Received in final form 11 May 2010, Accepted 17 May 2010)

\begin{abstract}
The electronic and magnetic properties of $\mathrm{Cu}$-doped $\mathrm{GaN}$ with a $\mathrm{Cu}$ concentration of $6.25 \%$ and $\mathbf{1 2 . 5 \%}$ are examined theoretically using the full-potential linear muffin-tin orbital method. The magnetic moment of $\mathrm{Cu}$ atoms decreases with increasing $\mathrm{Cu}$ concentration. The spin-polarization of $\mathrm{Cu}$ atoms is reduced due to the $\mathrm{Cu}$ d-d interaction depending on the distance between the nearest neighbouring $\mathrm{Cu}$ atoms. $\mathrm{Cu}$ atoms exhibits a clustering tendency in GaN. For $\mathrm{Cu}$-adsorbed $\mathrm{GaN}$ thin films with a surface coverage of 0.25 , the ferromagnetic state is found to be the energetically favourable state with an induced magnetic moment of $0.54 \mu_{\mathrm{B}}$ per supercell.
\end{abstract}

Keywords : nonmagnetic dopant, ferromagnetic ordering, thin film

\section{Introduction}

Group III-nitrides with magnetic dopants [1-9], such as 3d transition-metals of $\mathrm{V}, \mathrm{Cr}, \mathrm{Mn}, \mathrm{Fe}, \mathrm{Co}$, and $\mathrm{Ni}$, have attracted considerable interest due to their potential applications in spintronics devices that utilize both the charge and spin of electrons to create new functionalities beyond conventional semiconductors. Ideal diluted magnetic semiconductors (DMSs) should exhibit ferromagnetism at room temperature for practical applications and have a homogeneous distribution of magnetic dopants. However, the presence of magnetic precipitates in the host semiconductor in forms of clusters or secondary phases of magnetic impurities is detrimental to their real applications. For example, in Mn-doped GaN, clusters of $\mathrm{Mn}$ and secondary Ga-Mn and Mn-N phases have been observed $[10,11]$. In $\mathrm{Cr}$-doped $\mathrm{GaN}$, the magnetic moment of $\mathrm{Cr}$ in $\mathrm{GaN}$ was significantly smaller than the expected saturation value by $3 \mu_{\mathrm{B}}$, and varied in a wide range by $0.2-1.8 \mu_{\mathrm{B}}$ per atom [12-14].

The precipitates of nonmagnetic (NM) dopants do not contribute to the magnetic ordering. Recent theoretical investigations have reported the ferromagnetic ordering of normally NM materials in GaN [15-18]. For $4 d$-metal Pd

*Corresponding author: Tel: +82-43-261-3338

Fax: +82-43-274-7811, e-mail: kangsub@cbnu.ac.kr in $\mathrm{GaN}, \mathrm{Pd}$ orders ferromagnetically in $\mathrm{GaN}$ [15]. Clusters of Pd were found experimentally to be ordered ferromagnetically. Taniyama et al. reported that the magnetization of $\mathrm{Pd}$ increases rapidly with decreasing $\mathrm{Pd}$ particle size [19]. Cox et al. [20] have reported that Pd clusters with more than 12 atoms exhibit NM ordering. This shows that the magnetic moment is sizable with a small particle size $<10$ atoms. The size of a cluster has an effect on the spinpolarization of $\mathrm{Pd}$ atoms. Besides $\mathrm{Pd}$, it has been predicted that a $3 d$-metal $\mathrm{Cu}$ is a potential NM dopant for $\mathrm{GaN}$, even though more challenging theoretical and experimental investigations are needed. Wu et al. [16] reported that $\mathrm{Cu}$-doped $\mathrm{GaN}$ with $6.25 \%$ favors a ferromagnetic ground state. They showed that the spin-polarization of $\mathrm{Cu}$ and the neighboring $\mathrm{N}$ atoms can be explained in terms of $p$ - $d$ hybridization. In the case of $\mathrm{Cu}$-doped $\mathrm{ZnO}$, Ye et al. examined ferromagnetism by numerical calculations based on density functional theory [21]. They also predicted that the electronic states near the Fermi level form strong hybridization between the $\mathrm{Cu}$ dopant and neighboring $\mathrm{O}$ atoms. Interestingly, the local magnetic moment of $\mathrm{Cu}$ atoms is not dependent on the $\mathrm{Cu}$ concentration. However, for $\mathrm{Cu}$-doped $\mathrm{ZnO}$ thin films, the local magnetic moment decreased with increasing $\mathrm{Cu}$ concentration [22]. In this study, the possibility of $\mathrm{Cu}$ as a NM dopant to fabricate GaN-based DMS is evaluated by firstprinciples calculations based on spin density functional 
theory with the main focus on the spin-polarization of $\mathrm{Cu}$ and neighboring $\mathrm{N}$ atoms with increasing $\mathrm{Cu}$ concentration. In contrast to $\mathrm{Cu}$-doped $\mathrm{ZnO}$ [21], the spin-polarization of the $\mathrm{Cu}$ dopant in $\mathrm{GaN}$ decreases with increasing $\mathrm{Cu}$ concentration. The $\mathrm{Cu}$ dopant in $\mathrm{GaN}$ may form clusters or secondary $\mathrm{Ga}-\mathrm{Cu}$ or $\mathrm{Cu}-\mathrm{N}$ phases. As mentioned above, these precipitates do not contribute to the magnetism.

\section{Modeling and Calculations}

The electronic properties on $\mathrm{Cu}$-doped $\mathrm{GaN}$ at a concentration of 6.25 and $12.5 \%$ are investigated for supercells containing 32 atoms with one and two Ga atoms substituted by $\mathrm{Cu}$. Supercells of 72 atoms with one, two, three and four $\mathrm{Ga}$ atoms substituted by $\mathrm{Cu}$ are also examined, i.e. four doping levels are checked. In addition, a calculation of a thin film adsorbed $\mathrm{Cu}$ in the surface layer (by substitution) is performed. The electronic properties of the thin film are compared with those of $\mathrm{Ga}_{1-\mathrm{x}} \mathrm{Cu}_{\mathrm{x}} \mathrm{N}(x=0.0625$ and 0.125$)$, where applicable in our considered supercell. The wurtzite GaN substrate (thin film) is modeled by eight atomic layers, which are separated by six layers of vacuum. The atomic structure with one $\mathrm{Ga}$ atom substituted with $\mathrm{Cu}$ corresponds to a surface coverage of 0.25 .

The experimentally determined lattice constants of $\mathrm{GaN}$ in the wurtzite structure are used in the calculations, with $a=3.162 \AA, c=5.142 \AA$, and $u=0.377$ [23], where $u$ is the displacement of $\mathrm{Ga}$ and $\mathrm{N}$ sublattices along the $c$ axis. That is, the nearest neighboring distance is $0.377 c\left(=u^{*} c\right)$. The $c / a$ ratio of $\mathrm{GaN}$ is slightly larger than that reported previously [23]. The equilibrium lattice structure is expanded by $0.6 \%$ compared to the experimental one. The equilibrium lattice constants $a$ and $c$ of wurtzite GaN are 3.1809 and $5.1728 \AA$, respectively. This study is performed using the first-principles full-potential linear muffin-tin orbital (FPLMTO) method [24, 25] within both the local density approximation (LDA) and generalized gradient approximation (GGA) with the exchange-correlation functional proposed by the Janak-Moruzzi-Williams scheme [26]. The electron wave function is expanded in plane waves with a cut-off energy of 252.96 (224.40), 368.56 and 539.92 (477.36) eV for the $s, p$, and $d$ orbitals of $\mathrm{Ga}(\mathrm{Cu})$, respectively. The charge density is determined self-consistently using gamma-centered $4 \times 4 \times 4$ and $6 \times 6 \times 2$ grids, respectively, in the Brillouin zone for the bulk and thin film systems, which corresponds to 64 and $72 k$ points, respectively. Using the number of $k$ points ensures that the total energies and magnetic moments are converged within $10 \mathrm{meV} /$ cell and $0.01 \mu_{\mathrm{B}} /$ atom, respectively.

\section{Results and Discussion}

The ferromagnetic (FM) order of $\mathrm{Cu}$ atoms in $\mathrm{GaN}$ is more energetically favorable than theNM or antiferromagnetic (AFM) states. The ferromagnetic state is more energetically favorable than the AFM state, and its difference in energy is quite small $(31.5 \mathrm{meV})$ for a concentration of $6.25 \%$. The differences in the total energies between the FM and NM states at 6.25 and $12.5 \% \mathrm{Cu}$ are 73.5 and $108.8 \mathrm{meV}$, respectively. The magnetic energy gain in high concentrations is larger than that at lower concentrations. For a $\mathrm{Cu}$ concentration of $6.25 \%$, the equilibrium lattice constants changed slightly by $0.1 \%,-0.003 \AA$ for $a$ and $-0.005 \AA$ for $c$ after structural optimization. For a $\mathrm{Cu}$ concentration of $12.5 \%$, the equilibrium lattice constants changed by $-0.011 \AA$ and $-0.015 \AA$ for $a$ and $c$, respectively, compared to pure GaN. For pure GaN, the LDA calculation provides similar results to the experimental results ( $\sim 22.3 \AA^{3}$ in the unit-cell volume). However, shows the GGA overestimates the calculated equilibrium unitcell volume with a $23.8 \AA^{3}$ (Fig. 1). The volume of a supercell decreases with increasing $\mathrm{Cu}$ concentration. These decreases are attributed to the small difference in atomic radius between $\mathrm{Cu}$ and $\mathrm{Ga}$. Each $\mathrm{Cu}-\mathrm{N}$ bond length of the $\mathrm{CuN}_{4}$ tetrahedron is approximately $2.024 \AA$ with some structural distortion present. In the case of two $\mathrm{Cu}$ atoms, two cases of the $\mathrm{Cu}-\mathrm{Cu}$ distance with the tetrahedral structure are considered. That is, the $\mathrm{Cu}-\mathrm{Cu}$ distance is 3.1585 and $3.1778 \AA$ for two $\mathrm{Cu}$ atoms positioned along the $c$-axis and in a basal plane, respectively. The system with a $\mathrm{Cu}-\mathrm{Cu}$ distance of $3.1585 \AA$ in $\mathrm{GaN}$ is more energetically favorable than that of $3.1778 \AA$. The difference in the total energies between both systems with a Cu-Cu distance of 3.1585 and $3.1778 \AA$ is $346 \mathrm{meV}$ per supercell. Therefore, there is a tendency for $\mathrm{Cu}$ clustering

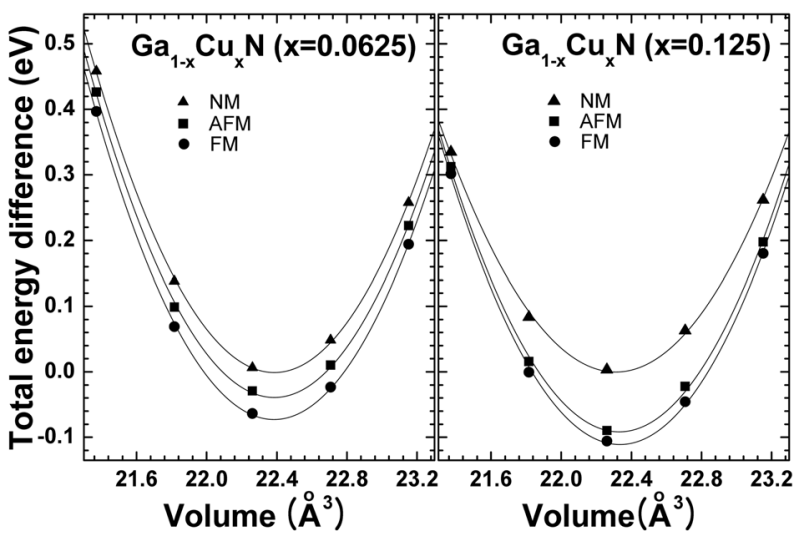

Fig. 1. Total energy differences of the super cell containing $6.25 \%$ and $12.5 \% \mathrm{Cu}$ with respect to the reference $\mathrm{NM}$ state as a function of the unit-cell volume $\left(\AA^{3}\right)$. 
Table 1. Magnetic moments $\left(\mu_{\mathrm{B}}\right)$ and band-splitting energies of impurity atoms $(\Delta \mathrm{E}, \mathrm{eV})$ for a thin film with a surface coverage $(\theta)$ of 0.25 and $\mathrm{Ga}_{1-\mathrm{x}} \mathrm{Cu}_{\mathrm{x}} \mathrm{N}$. Cu1 or $\mathrm{Cu} 2$ denotes the occupied $\mathrm{Cu}$ site. The results are compared with those of other previous calculations (parenthesis).

\begin{tabular}{|c|c|c|c|c|}
\hline \multirow{3}{*}{ Magnetism } & \multirow{3}{*}{$\begin{array}{c}\text { Thin film } \\
\text { Surface } \\
\text { FM }\end{array}$} & \multicolumn{3}{|c|}{ Two doping level } \\
\hline & & $\mathrm{Cul}$ & $\mathrm{Cul}$ & $\mathrm{Cu} 2$ \\
\hline & & FM/AFM & FM/AFM & FM/AFM \\
\hline $\mathrm{Cu}$ & 0.27 & $0.67(0.70)^{\mathrm{a} /} / 0.54$ & $0.06 / 0.21$ & $0.13 / 0.27$ \\
\hline $\mathrm{N}\left(\right.$ in $\left.\mathrm{CuN}_{4}\right)$ & $(\theta=0.25)$ & $(x=0.0625)$ & \multicolumn{2}{|c|}{$(x=0.125)$} \\
\hline Top site & - & $0.18(0.22)^{\mathrm{a} / 0.12}$ & $0.02 / 0.03$ & $0.03 / 0.03$ \\
\hline Basal site & 0.09 & $0.20(0.25)^{\mathrm{a}} / 0.14$ & $0.03 / 0.06$ & $0.04 / 0.07$ \\
\hline$\Delta \mathrm{E}$ & 0.301 & $0.731 / 0.639$ & $0.068 / 0.238$ & $0.108 / 0.234$ \\
\hline
\end{tabular}

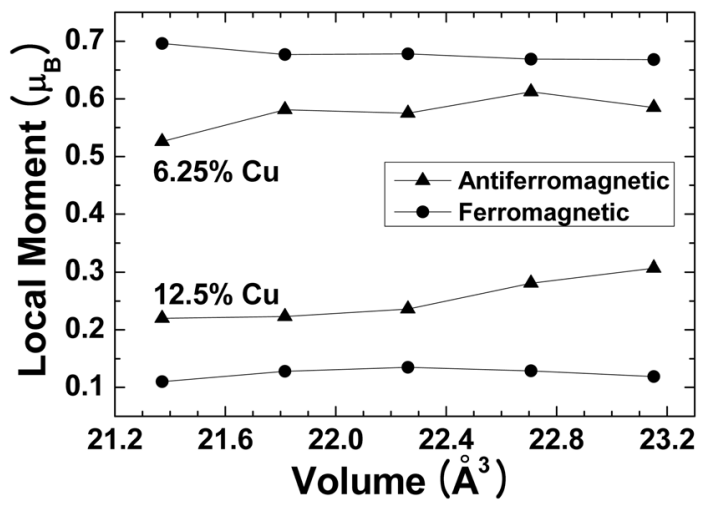

Fig. 2. The magnetic moment of $\mathrm{Cu}$ atoms in $\mathrm{Cu}$-doped $\mathrm{GaN}$ containing $6.25 \%$ and $12.5 \% \mathrm{Cu}$ as a function of the unit-cell volume $\left(\AA^{3}\right)$.

in $\mathrm{Cu}$-doped $\mathrm{GaN}$. A Cu-Cu distance of $3.1778 \AA$ corresponds to a lattice parameter of $a$. This is slightly different from the electronic structure each other. In Table 1, the two sites are represented as $\mathrm{Cu} 1$ or $\mathrm{Cu} 2$ (see Fig. 3). The four $\mathrm{N}$ sites and $\mathrm{Cu} 1$ or $\mathrm{Cu} 2$ site have the structure of a distorted $\mathrm{CuN}_{4}$ tetrahedron. The magnetic moments of these sites are listed in Table 1, and are compared with those of the thin film.

For the $\mathrm{Cu}$ concentration of $6.25 \%$ with the FM state, the $\mathrm{Cu}$ atom is found to be the main contributor to the magnetic moment with a localized magnetic moment of $0.67 \mu_{\mathrm{B}}$ per atom. The four surrounding $\mathrm{N}$ atoms in the $\mathrm{CuN}_{4}$ tetrahedron are polarized with a magnetic moment of $0.18 \mu_{\mathrm{B}}$ for the top $\mathrm{N}$ site and $0.20 \mu_{\mathrm{B}}$ for the other three $\mathrm{N}$ sites in the basal plane. The smaller magnetic moment for the top site $\mathrm{N}$ is due to a distortion of the tetrahedron, i.e. the $\mathrm{Cu}-\mathrm{N}$ bond is smaller for the top site $\mathrm{N}$ than the three lying in the basal plane. This is also because the spin-polarization of the $\mathrm{Ga}$ and other $\mathrm{N}$ atoms away from the $\mathrm{CuN}_{4}$ tetrahedron is very small. At a concentration of $12.5 \%$, the total magnetic moment per supercell in the ground state is $0.48 \mu_{\mathrm{B}}$, or $0.06 \mu_{\mathrm{B}}$ or 0.13 $\mu_{\mathrm{B}}$ per $\mathrm{Cu}$ atom. The nearest neighboring four $\mathrm{N}$ atoms are polarized with average magnetic moments of $0.03 \mu_{\mathrm{B}}$ per $\mathrm{N}$ atom. The FM state is more energetically favorable than the other states. The local magnetic moment for the FM state is almost constant regardless of the lattice parameter, whereas it increases slightly for the AFM state, as shown in Fig. 2. The spin-polarization of the supercell decreases with increasing $\mathrm{Cu}$ concentration. This is due to $3 d-3 d$ hybridization by the $\mathrm{Cu}-\mathrm{Cu}$ interaction depending on the distance between the nearest-neighbor $\mathrm{Cu}$ atoms. At low dopant concentrations, the $\mathrm{Cu}$ magnetic moment of the FM state is larger than that of AFM state. On the other hand, for a high concentration, the $\mathrm{Cu}$ magnetic moment of the AFM state is slightly larger than that of the FM state. The magnetic property of $\mathrm{Cu}$ in $\mathrm{GaN}$ depends on the dopant concentration. The magnetic moment of $\mathrm{Cu}$ atom decreases with increasing concentration.

The calculations are repeated using a supercell with one, two, three and four substitutional $\mathrm{Cu}$ atoms, which corresponds to a concentration of $2.78,5.55,8.33$ and $11.11 \%$, respectively. In the case of $5.55 \% \mathrm{Cu}$, the supercell consists of 72 atoms with two Ga atom substituted by $\mathrm{Cu}$. The electronic and magnetic properties of the super-

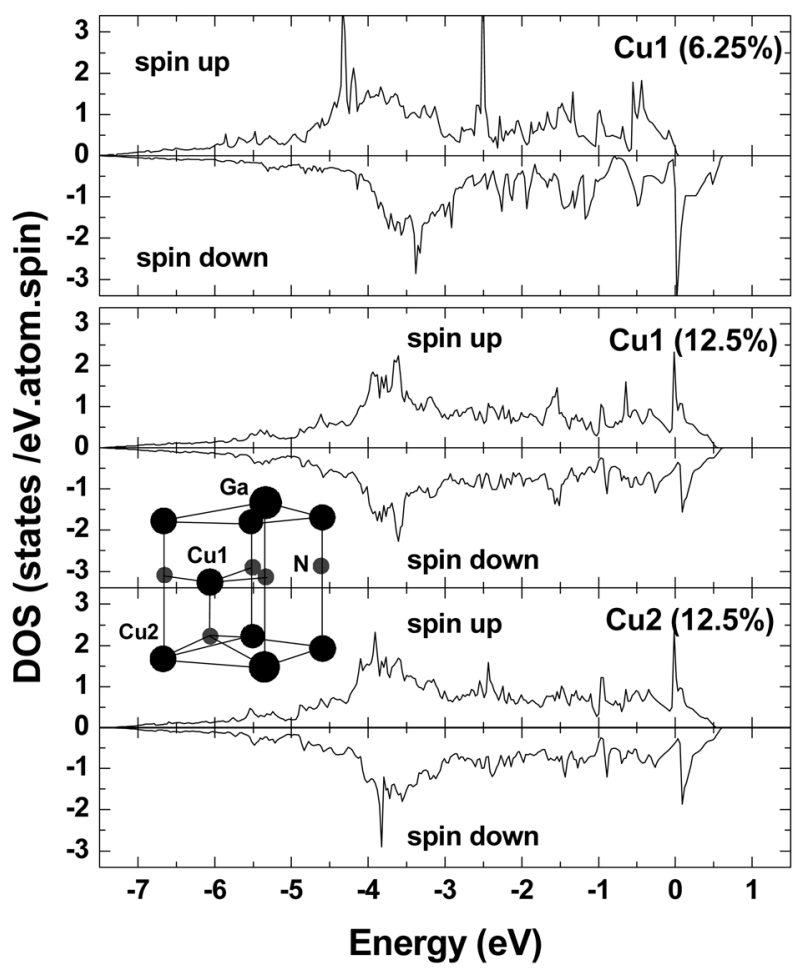

Fig. 3. Projected DOS for $\mathrm{Cu}$ in $\mathrm{Ga}_{0.9375} \mathrm{Cu}_{0.0625} \mathrm{~N}$ and $\mathrm{Ga}_{0.875} \mathrm{Cu}_{0.125} \mathrm{~N}$. The Fermi level is set to zero. The atomic sites of $\mathrm{Cu} 1$ and $\mathrm{Cu} 2$ are shown in the inset. 
cell are similar to those of the system consisting of 32 atoms with one $\mathrm{Ga}$ atom substituted by $\mathrm{Cu}$. For the equilibrium atomic volume of these calculations, all NM and FM phases range from 21.37 to $23.15 \AA^{3}$, corresponding to an equilibrium Wigner-Seitz radius $r_{W S} \sim$ 2.69 a.u..

The electronic configuration of $\mathrm{Cu}$ and $\mathrm{N}$ atoms is $4 \mathrm{~s}^{0.42} 4 \mathrm{p}^{0.42} 3 \mathrm{~d}^{9.00}$ and $2 \mathrm{~s}^{1.11} 2 \mathrm{p}^{2.26}$ within the muffin tin sphere, respectively. A charge of $1.16 e(1.63 e)$ per $\mathrm{Cu}(\mathrm{N})$ site moves into the interstitial region. The charge configuration of $\mathrm{N} \mathrm{p}$ electrons is not changed when there is another $\mathrm{Cu}$ atom near a $\mathrm{Cu}$ atom, whereas the electronic density of the $3 \mathrm{~d}$ state in each $\mathrm{Cu}$ site changes slightly due to the $\mathrm{Cu}-\mathrm{Cu}$ interaction and difference in the nearest neighboring $\mathrm{Cu}-\mathrm{N}$ distance. The charge configuration changes to a repulsive interaction between $\mathrm{Cu} 3 \mathrm{~d}$ and $\mathrm{N}$ $2 p$ electrons. Accordingly, the spin-polarization of $\mathrm{Cu} 3 \mathrm{~d}$ electrons is reduced. The magnetic moment of $\mathrm{Cu}$ atoms should decrease with increasing $\mathrm{Cu}$ concentration.

Fig. 3 shows the projected densities of states (DOS) for $\mathrm{Ga}_{0.9375} \mathrm{Cu}_{0.0625} \mathrm{~N}$ and $\mathrm{Ga}_{0.875} \mathrm{Cu}_{0.125} \mathrm{~N}$. The introduction of

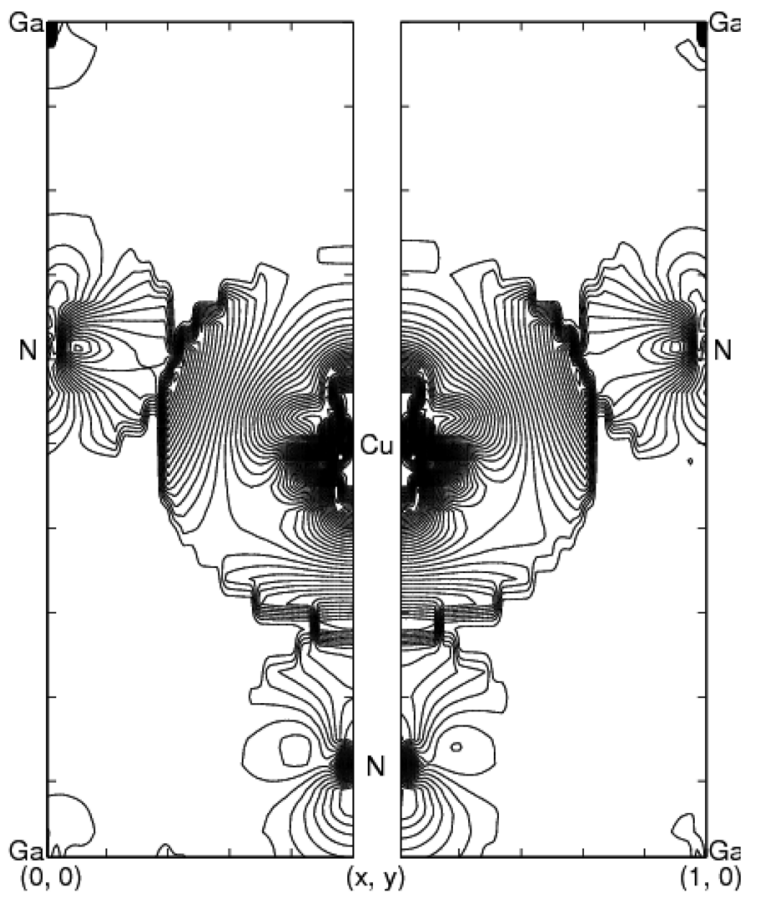

Fig. 4. The charge density difference $(\Delta \rho(r, \mathrm{R}))$ for $\mathrm{Ga}_{0.9375^{-}}$ $\mathrm{Cu}_{0.0625} \mathrm{~N}$ in the ground state. $\Delta \rho(\mathrm{r}, \mathrm{R})$ is defined as $\rho^{\mathrm{Cu}}{ }_{\mathrm{GaN}}(\mathrm{r}, \mathrm{R})-$ $\rho_{\mathrm{GaN}}(\mathrm{r}, \mathrm{R})-\rho^{\mathrm{Cu}}(\mathrm{r}, \mathrm{R})$, where $\rho^{\mathrm{Cu}}{ }_{\mathrm{GaN}}(\mathrm{r}, \mathrm{R})$ is the total charge density, $\rho_{\mathrm{GaN}}(\mathrm{r}, \mathrm{R})$ and $\rho^{\mathrm{Cu}}(\mathrm{r}, \mathrm{R})$ are the charge densities for $\mathrm{GaN}$ with a empty site (point defect of $\mathrm{Ga}$ site) and isolated one $\mathrm{Cu}$ atom, respectively. The contour is shown on the plane of $(1120)$ with intervals a $2.0 \times 10^{-4} \mathrm{e} /(\text { a.u. })^{3}$. The coordinate of $(\mathrm{x}, \mathrm{y})$ corresponds to $(1 / 2, \sqrt{3} / 6)$. Here, the lattice parameter of $a$ is set to 1 .
$\mathrm{Cu}$ in $\mathrm{GaN}$ shows a slight upward shift in energy by 0.35 $\mathrm{eV}$ up to the Fermi level (majority-band). The electrons of the dopant are spin-polarized with a splitting-energy of $0.731 \mathrm{eV}$ per $\mathrm{Cu}$ atom. The unoccupied minority-band maximum is located at approximately $1 \mathrm{eV}$. The $\mathrm{Cu}$ band is formed by the $\mathrm{Cu} 3 \mathrm{~d}$ level hybridizing with the $2 \mathrm{p}$ levels of the $\mathrm{N}$ neighbors of $\mathrm{Cu}$. In the case of a thin film, the number of neighboring $\mathrm{N}$ atoms on the surface $\mathrm{Cu}$ site is lower than that of $\mathrm{Cu}$-doped $\mathrm{GaN}$. Therefore, the interaction between the $\mathrm{Cu} 4 \mathrm{~d}$ and $\mathrm{N} 2 \mathrm{p}$ states is weak. The energies of spin-splitting between their atoms are low and the spin-polarization is reduced. The band gap of pure $\mathrm{GaN}$ thin film is narrowed by the surface states. The $\mathrm{Cu}$ magnetic moment is decreased by $0.27 \mu_{\mathrm{B}}$ per $\mathrm{Cu}$ atom. This result is compared with previous work [27]. This is a similar trend to that of $\mathrm{Cu}$-doped $\mathrm{ZnO}$ thin films with magnetic moment of $0.4 \mu_{\mathrm{B}}$ per $\mathrm{Cu}$ atom [22].

Fig. 4 shows the charge density of $\mathrm{Ga}_{0.9375} \mathrm{Cu}_{0.0625} \mathrm{~N}$ within the FM state, and can be compared with the interaction between $\mathrm{Ga}, \mathrm{Cu}$, and $\mathrm{N}$ atoms. A strong $\mathrm{Cu}-\mathrm{N}$ interaction occurs by large charge accumulation between neighboring $\mathrm{Cu}$ and $\mathrm{N}$ atoms. The cohesive energy of one $\mathrm{Cu}$ atom in $\mathrm{GaN}$ is $-8.99 \mathrm{eV}$. The cohesive energy is defined as follows: $E_{t o t}(G a, C u, N)-E_{t o t}(G a, N)-E_{t o t}(C u)$, where $E_{\text {tot }}(\mathrm{Ga}, \mathrm{Cu}, \mathrm{N})$ is the total energy of $\mathrm{Cu}$-doped system, $E_{t o t}(G a, N)$ is the total energy of pure $\mathrm{GaN}$, and $E_{\text {tot }}(\mathrm{Cu})$ is that of an isolated $\mathrm{Cu}$ atom. The exchange splitting-energy between the spin-down and up bands of $\mathrm{Cu} 4 s$ and $3 d$ are $0.408 \mathrm{eV}$ and $0.323 \mathrm{eV}$, respectively. The $\mathrm{N}$ magnetic moments by an interaction of $\mathrm{Cu}-\mathrm{N}$ atoms are sizable. The peak at $-4.36 \mathrm{eV}$ of $\mathrm{Cu}-3 d$ in the majority spin-channel overlaps that of $\mathrm{N}-2 p$ at the $\mathrm{CuN}_{4}$ tetrahedron. The peak at $-0.56 \mathrm{eV}$ of $\mathrm{Cu}-3 d$ also overlaps with that of $\mathrm{N}-2 p$. In the minority spin-channel, the $2 p$ state of the four connecting $\mathrm{N}$ atoms contributes significantly to the unoccupied states. These characteristics indicate strong hybridization between $\mathrm{Cu}$ and its four neighboring $\mathrm{N}$ atoms. The $\mathrm{Cu}$ atom hybridizes strongly with the neighboring anions of GaN. Thus, the neighboring anions are spin-polarized with large (relatively) magnetization in the same order as that of the dopant. The $\mathrm{Cu}-\mathrm{N}$ interaction has a strong effect on $\mathrm{N}$ magnetization by the presence of $\mathrm{Cu}$ neighbors with a low concentration. However, the $\mathrm{Cu}$ local moment should disappear as the $\mathrm{Cu}$ concentration increases.

\section{Conclusions}

This study examined the electronic and magnetic properties derived from the band structure calculations for a thin film and $\mathrm{Cu}$-doped wurtzite $\mathrm{GaN}$ using the FPLMTO 
method. The NM dopant at low concentrations becomes spin-polarized when incorporated substitutionally into GaN. The FM state is more energetically favorable than the AFM or NM states. FM ordering of the dopant and four neighboring $\mathrm{N}$ atoms is produced by strong $p$ - $d$ hybridization. However, the magnetic moment per $\mathrm{Cu}$ atom decreases with increasing $\mathrm{Cu}$-doping concentration. The reduced magnetic moment is due to an increase in the number of $\mathrm{Cu}$ atoms occupying the adjacent cation lattice positions.

\section{Acknowledgements}

This study was supported by the research fund of Academic Research Program in 2009 at Chungbuk National University.

\section{References}

[1] Masahiko Hashimoto, Yi-Kai Zhou, Masahito Kanamura, Hajime Asahi, Solid State Comm. 122, 37 (2002).

[2] D. O'Mahony, J. G. Lunney, G. Tobin, and E. McGlynn, Solid-State Electronics 47, 533 (2003).

[3] K. Sato, P. H. Dederichs, and H. Katayama-Yoshida, J. Kudrnovsky, Physica B 340, 863 (2003).

[4] B. S. Kang, W. C. Kim, Y. Y. Shong, and H. J. Kang, J. Crystal Growth 287, 74 (2006).

[5] ByungSub Kang, HaengKi Lee, KyeongSup Kim, and HeeJae Kang, Phys. Scr. 79, 025701 (2009).

[6] Q. Wang, A. K. Kandalam, Q. Sun, and P. Jena, Phys. Rev. B 73, 115411 (2006).

[7] R. M. Frazier, G. T. Thaler, J. Y. Leifer, J. K. Hite, B. P. Gila, C. R. Abernathy, and S. J. Pearton, Appl. Phys. Lett. 86, 052101 (2005).

[8] A. Ney, R. Rajaram, S. S. P. Parkin, T. Kammermeier, and S. Dhar, Phys. Rev. B 76, 035205 (2007).

[9] X. Y. Cui, D. Fernandez-Hevia, B. Delley, A. J. Freeman, and C. Stampfl, J. Appl. Phys. 101, 103917 (2007).

[10] J. M. Baik, H. W. Jong, J. K. Kim, and J. L. Lee, Appl.
Phys. Lett. 82, 583 (2003).

[11] S. Dhar, O. Brandt, A. Trampert, L. Daweritz, K. J. Friedland, K. H. Ploog, J. Leller, B. Beschoten, and G. Guntherodt, Appl. Phys. Lett. 82, 2077 (2003).

[12] H. X. Lju, S. Y. Wu, R. K. Singh, L. Gu, D. Smith, N. Newman, N. R. Dilley, L. Montes, and M. B. Simmonds, Appl. Phys. Lett. 85, 4076 (2001).

[13] A. Y. Polyakov, N. B. Simirnov. A. V. Govorkov, N. V. Pashkova, A. A. Shlensky, S. J. Pearton, M. E. Overberg, C. R. Abernathy, J. M. Zavada, and R. G. Wilson, J. Appl. Phys. 93, 5388 (2003).

[14] X. Y. Cui, J. E. Medvedeva, B. Delly, A. J. Freeman, and C. Stampfl, Phys. Rev. Lett. 95, 256404 (2005).

[15] K. Osuch, E. B. Lombardi, and L. Adamowicz, Phys. Rev. B 71, 165213 (2005).

[16] R. Q. Wu, G. W. Peng, L. Liu, and Y. P. Feng, Z. G. Huang, and Q. Y. Wu, Appl. Phys. Lett. 89, 062505 (2006).

[17] L. Kronix, M. Jain, and J. R. Chelikowsky, Phys. Rev. B 66, 041203(R) (2002).

[18] B. Sampedro, P. Crespo, A. Hernando, R. Litráan, J. C. Sánchez López, C. López Cartes, A. Fernandez, J. Ramirez, J. González Calbet, and M. Vallet, Phys. Rev. Lett. 91, 237203 (2003).

[19] T. Taniyama, E. Ohta, and T. Sato, Europhys. Lett. 38, 195 (1997).

[20] A. J. Cox, J. G. Louderback, S. E. Apsel, and L. A. Bloomfield, Phys. Rev. B 49, 12295 (1994).

[21] Lin-Hui Ye, A. J. Freeman, and B. Delley, Phys. Rev. B 73, 033203 (2006).

[22] D. B. Buchholz, R. P. H. Chang, J. H. Song, and J. B. Ketterson, Appl. Phys. Lett. 87, 082504 (2005).

[23] A. F. Wright, J. F. Nelson, Phys. Rev. B 51, 7866 (1995).

[24] W. Kohn and L. J. Sham, Phys. Rev. 140, A1133 (1965).

[25] S. Y. Savrasov, Phys. Rev. B 54, 16470 (1996), and references therein.

[26] J. F. Janak, V. L. Moruzzi, and A. R. Williams, Phys. Rev. B 12, 1257 (1975).

[27] Peng-Wei Wang, Xue-Jin Zhang, Bai-Qi Wang, XinZheng Zhang, and Da-Peng Yu, Chinese Phys. Lett. 25, 3040 (2008). 